\title{
IMPEDANCE OF PERIODIC IRISES IN A BEAM PIPE*
}

\author{
Shicheng Jiang and Robert L. Gluckstern \\ Physics Department, University of Maryland, College Park, MD 20742, USA \\ Hiromi Okamoto \\ Institute for Chemical Research, Kyoto University, Kyoto 611, Japan
}

\begin{abstract}
In a recent paper[1], we constructed a variational form to calculate both the longitudinal and transverse impedance of a thick iris in a beam pipe. Implementation of this calculation led to rapidly converging and accurate values for these impedances for a circular beam pipe and iris. We have now constructed an analogous variational form for the longitudinal and transverse impedance of periodic irises in a beam pipe, with similar convergence and accuracy properties. In this case the numerically calculated impedance is imaginary, except for isolated narrow resonances corresponding to modes propagating with the velocity of light. The real part of impedance is obtained by using causality. Analysis and numerical results are discussed and presented.
\end{abstract}

\section{Introduction}

In a previous publication[1], we calculated both the longitudinal and transverse coupling impedance of an iris in a beam pipe. In particular we constructed a variational formulation for the impedance, where the trial function was the transverse electric fields at the two junctions of the beam pipe and the iris. The numerical implementation of this formulation proved to be extremely well convergent for a circular beam pipe and iris, requiring only a few terms in the expansion of the trial functions in terms of TM and TE transverse modes in the iris region.

In this work, we construct a corresponding variational formulation for the longitudinal and transverse impedance of a beam pipe loaded with periodic irises. The numerical implementation for a circular beam pipe with periodic circular irises is again well convergent. The numerically calculated impedance in this case is imaginary, except for isolated narrow resonances corresponding to modes propagating with the velocity of light. However, the real part of the impedance is retrieved from the information the imaginary part carries, in the form of a sum of delta functions.

\section{Analysis}

We will consider a beam pipe of arbitrary cross section loaded with periodic irises of arbitrary cross section, both homogeneous in the axial direction. The planes involving the iris side walls are perpendicular to the beam pipe axis. We denote the period of irises as $L$, and the width of irises as $g$. We consider the $m t h$ iris and its following pipe cavity as the $m t h$ cell of length $L$. We set the coordinate origin at the center of the zeroth iris and the axis of the pipe as the $z$ axis. The cross sectional area of the iris hole is denoted by $S_{1}$ while $S_{2}$ represents the side walls of the iris.
Both in the iris hole and in the pipe cavity, the electromagnetic fields are superpositions of source fields and pipe fields which can be expanded as sums over the normal modes in relevant regions. We use Latin letters as the subscripts of the quantities defined in the pipe cavity, and Greek letters for those defined in the iris hole. The time dependent factor $\exp (j \omega t)$ attached to all fields is omitted for simplicity.

In the pipe cavity region, the source fields are generated by the ultrarelativistic charged particle beam moving near or along the axis of a smooth pipe. Specifically, in the frequency domain we have:

$$
\mathrm{E}_{0}=Z_{0} \mathrm{H}_{0} \times \hat{z}=A_{0} e^{-j k z} \nabla \perp \chi,
$$

where $Z_{0}$ is the free-space impedance, $A_{0}$ is a constant, and $\hat{z}$ is unit vector in positive $z$ direction. $\nabla_{\perp}$ is defined as $\nabla_{\perp}=$ $\hat{\mathrm{x}} \frac{\partial}{\partial x}+\hat{\mathrm{Y}} \frac{\partial}{\partial y}$. The source field potential $\chi$ is a function of transverse coordinates which satisfies the boundary conditions on the pipe surface.

The pipe fields contain both left and right traveling waves with wave number $\beta_{n}$. Floquet's theorem says that in a periodic structure the electromagnetic fields in the $m t h$ cell must be identical to those in the $(m+1) t h$ cell except for a constant phase factor. We then write:

$$
\begin{gathered}
\frac{\mathrm{E}_{\perp}}{A_{0}}=e^{-j k z} \nabla \perp \\
\quad+\sum_{n} e_{n} e^{-j k m L}\left[A_{n} e^{-j \beta_{n}(z-m L)}\right. \\
\left.+B_{n} e^{j \beta_{n}(z-m L)}\right], \\
\frac{Z_{0} \mathrm{H} \perp \times \hat{z}}{A_{0}}=e^{-j k z} \nabla \perp \chi+\sum_{n} e_{n} \lambda_{n} e^{-j k m L}\left[A_{n} e^{-j \beta_{n}(z-m L)}\right. \\
\left.-B_{n} e^{j \beta_{n}(z-m L)}\right] .
\end{gathered}
$$

Here, $e_{n}$ are the transverse electric normal modes defined on the cross section of the pipe region (Its area is $S_{1}+S_{2}$.), that means $\int_{S_{1}+S_{2}} e_{n} \cdot e_{n^{\prime}} d S=\delta_{n n^{\prime}}, A_{n}$ and $B_{n}$ are expansion coefficients, and $\lambda_{n}$ is $k / \beta_{n}$ for TM modes, $\beta_{n} / k$ for TE modes.

In the iris hole, the source field potential is replaced by $\sigma$ which satisfies the boundary conditions on the hole surface, and we write:

$$
\begin{gathered}
\frac{\mathrm{E}_{\perp}}{A_{0}}=e^{-j k z} \nabla \perp \sigma+\sum_{\mu} \mathrm{e}_{\mu} e^{-j k m L}\left[C_{n} e^{-j \beta_{\mu}(z-m L)}\right. \\
\left.+D_{n} e^{j \beta_{\mu}(z-m L)}\right], \\
\frac{Z_{0} \mathrm{H} \perp \times \hat{\mathrm{z}}}{A_{0}}=e^{-j k z} \nabla \perp \sigma+\sum_{\mu} \mathrm{e}_{\mu} \lambda_{\mu} e^{-j k m L}\left[C_{\mu} e^{-j \beta_{\mu}(z-m L)}\right. \\
\left.-D_{n} e^{j \beta_{\mu}(z-m L)}\right] .
\end{gathered}
$$


Here, $e_{\mu}$ are the transverse electric normal modes defined on the cross section of the iris hole(Its area is $S_{1}$.), that means $\int_{S_{1}} e_{\mu}$. $\mathrm{e}_{\mu^{\prime}} d S=\delta_{\mu \mu^{\prime}}, C_{\mu}$ and $D_{\mu}$ are expansion coefficients, $\beta_{\mu}$ is the wave number in the iris hole, and $\lambda_{\mu}$ is $k / \beta_{\mu}$ for TM modes, $\beta_{\mu} / k$ for TE modes.

By introducing two unknown fields $\mathrm{U}$ and $\mathrm{V}$ defined as the transverse electric fields on $S_{1}$, which can be expanded in terms of $e_{\mu}$, and matching electromagnetic fields at the junctions of irises and pipe, we eventually get two integral equations:

$$
\begin{aligned}
& \int_{S_{1}} \stackrel{\leftrightarrow}{\mathrm{K}}_{11} \cdot \mathrm{U} d S+\int_{S_{1}} \stackrel{\leftrightarrow}{\mathrm{K}}_{12} \cdot \mathrm{V} d S=\mathrm{G}_{U}, \\
& \int_{S_{1}} \stackrel{\leftrightarrow}{\mathrm{K}}_{12} \cdot \mathrm{U} d S+\int_{S_{1}} \stackrel{\leftrightarrow}{\mathrm{K}}_{22} \cdot \mathrm{V} d S=\mathrm{G}_{V} .
\end{aligned}
$$

Here, $\stackrel{\leftrightarrow}{\mathrm{K}}_{11}, \stackrel{\leftrightarrow}{\mathrm{K}}_{12}$ and $\stackrel{\leftrightarrow}{\mathrm{K}}_{22}$ are tensor integral kernels:

$$
\begin{gathered}
\stackrel{\leftrightarrow}{\mathrm{K}}_{11}=\sum_{n} \mathrm{e}_{n} \mathrm{e}_{n}^{\prime} \frac{2 \lambda_{n}}{j \sin \beta_{n}(L-g)}\left[\cos k(L-g)-\cos \beta_{n}(L-g)\right] \\
\quad+\sum_{\mu} \mathrm{e}_{\mu} \mathrm{e}_{\mu}^{\prime} \frac{2 \lambda_{\mu}}{j \sin \beta_{\mu} g}\left[\cos k g-\cos \beta_{\mu} g\right] \\
\stackrel{\leftrightarrow}{\mathrm{K}}_{12}=\sum_{n} \mathrm{e}_{n} \mathrm{e}_{n}^{\prime} \frac{2 \lambda_{n} \sin k(L-g)}{\sin \beta_{n}(L-g)}+\sum_{\mu} \mathrm{e}_{\mu} \mathrm{e}_{\mu}^{\prime} \frac{2 \lambda_{\mu} \sin k g}{\sin \beta_{\mu} g}, \\
\stackrel{\leftrightarrow}{\mathrm{K}}_{22}=\sum_{n} \mathrm{e}_{n} \mathrm{e}_{n}^{\prime} \frac{2 \lambda_{n}}{j \sin \beta_{n}(L-g)}\left[\cos k(L-g)+\cos \beta_{n}(L-g)\right] \\
\quad+\sum_{\mu} \mathrm{e}_{\mu} \mathrm{e}_{\mu}^{\prime} \frac{2 \lambda_{\mu}}{j \sin \beta_{\mu} g}\left[\cos k g+\cos \beta_{\mu} g\right]
\end{gathered}
$$

And $\mathrm{G}_{U}$ and $\mathrm{G}_{V}$ are respectively:

$$
\begin{gathered}
\mathrm{G}_{U}=\sum_{n} \mathrm{e}_{n} \frac{2 \lambda_{n}\left(\rho_{n}+\chi_{n}\right)}{j \sin \beta_{n}(L-g)}\left[\cos k(L-g)-\cos \beta_{n}(L-g)\right], \\
\mathrm{G}_{V}=\sum_{n} \mathrm{e}_{n} \frac{2 \lambda_{n}\left(\rho_{n}+\chi_{n}\right) \sin k(L-g)}{\sin \beta_{n}(L-g)}-2 \nabla \perp \rho,
\end{gathered}
$$

where $\chi_{n}=\int_{S_{2}} \nabla \perp \chi \cdot e_{n} d S, \rho_{n}=\int_{S_{1}} \nabla \perp \rho \cdot e_{n} d S$, and $\rho$ is defined as $\rho=\chi-\sigma$.

Finally, the impedance of one cell of this periodic structure can be expressed as

$$
Z(k)=G_{0}(k)+Z_{v a r}(k),
$$

where the explicit term $G_{0}(k)$ is a sum over the indeces of the normal modes in the pipe region, and $Z_{v a r}(k)$ has been put into the variational form:

$$
Z_{v a r}(k)=\frac{\left[\int_{S_{1}} \mathrm{G}_{U} \cdot \mathrm{Ud} S+\int_{S_{1}} \mathrm{G}_{V} \cdot \mathrm{V} \mathrm{dS}\right]^{2}}{M},
$$

where $M$ is

$$
\begin{gathered}
M=\int_{S_{1}} d S \int_{S_{1}} d S^{\prime} \mathrm{U} \cdot \stackrel{\leftrightarrow}{\mathrm{K}}_{11} \cdot \mathrm{U}+2 \int_{S_{1}} d S \int_{S_{1}} d S^{\prime} \mathrm{U} \cdot \stackrel{\leftrightarrow}{\mathrm{K}}_{12} \cdot \mathrm{V} \\
+\int_{S_{1}} d S \int_{S_{1}} d S^{\prime} \mathrm{V} \cdot \stackrel{\leftrightarrow}{\mathrm{K}}_{22} \cdot \mathrm{V}
\end{gathered}
$$

\section{Numerical Results}

As an example, we use the formula to calculate the longitudinal and transverse impedance of a circular pipe loaded with circular irises. We obtain results for a wide range of structure parameters. We find that our computer code is well convergent over a large range of frequency for those structures. The calculated impedance is imaginary, except for isolated narrow resonances. The positions of the resonances are the intersections of dispersion curves with the light line. These are the resonant modes propagating with the velocity of light in the periodic structure. Fig. 1 presents the longitudinal impedance for a sample structure(The radius of beam pipe is $a$, the radius of iris hole is $b$. In our example, the ratios of $b / a=0.45, L / a=1.1$, and $g / a=0.35$.). The resonant modes and loss factors obtained from our calculation agree well with those from the well known computer codes KN7C and TRANSVRS [2].

\section{Discussion}

The missing real part of the impedance can be retrieved from the imaginary part by using causality. Taking the longitudinal case as an example(Note: $Z=R+j X$.),

$$
\frac{X_{\|}(k)}{Z_{0}}=\sum_{n} \frac{a_{n} k}{k_{n}^{2}-k^{2}},
$$

where all $a_{n}$ 's are greater than zero to satisfy Foster's Reactance Theorem[3]. If we now move the poles to a position slightly above the real axis, we can write the impedance for very small $\epsilon$. We use the relation $Z_{\|}(-k)=Z_{\|}^{*}(k)$ and write the imaginary part in the form:

$$
\frac{Z_{\|}(k)}{Z_{0}}=\sum_{n} \frac{j a_{n} k}{k_{n}^{2}-k^{2}+j \epsilon} .
$$

By using $\lim _{\epsilon \rightarrow 0}\left[\epsilon /\left(\epsilon^{2}+x^{2}\right)\right]=\pi \delta(x)$, we get

$$
\begin{aligned}
\frac{R_{\|}(k)}{Z_{0}} & =\lim _{\epsilon \rightarrow 0} \sum_{n} \frac{a_{n} k \epsilon}{\left(k_{n}^{2}-k^{2}\right)^{2}+\epsilon^{2}} \\
= & \pi \sum_{n} a_{n} k_{n} \delta\left(k^{2}-k_{n}^{2}\right) .
\end{aligned}
$$

We can therefore use the computed behavior of $X_{\|}(k)$ near each resonance at $k=k_{n}$ to determine $a_{n}$ by fitting $Z_{0} / X_{\|}(k)$ as a linear function of $k-k_{n}$ near $k=k_{n}$, and from these $a_{n}$ 's to obtain the real part of the impedance as a sum of delta functions with specified coefficient. Clearly these delta functions will be broadened if the wall conductivity is finite. Fig. 2 presents the delta functions of real part longitudinal impedance[4]. The first 10 resonances out of the 60 shown in Figs. 1 and 2 are identified in Table 1.

It appears that the resonances at $k=k_{n}$ correspond to the frequencies at which propagation of azmuthally symmetric modes through the structure produces a phase advance of $k L$ per period of length $L$, in order to be in synchronism with the particle moving with velocity $c$. This is confirmed from the dispersion curves calculated with our geometry, using the program KN7C.

The total longitudinal impedance is therefore

$$
\frac{Z_{\|}(k)}{Z_{0}}=\pi \sum_{n} a_{n} k_{n} \delta\left(k^{2}-k_{n}^{2}\right)+j k \sum_{n} \frac{a_{n}}{k_{n}^{2}-k^{2}},
$$


where the sum is over all values of $n$ corresponding to positive $k_{n}$. Clearly $Z_{\|}(-k)=Z_{\|}^{*}(k)$, as required.

A similar situation exists for the transverse impedance, where we find,

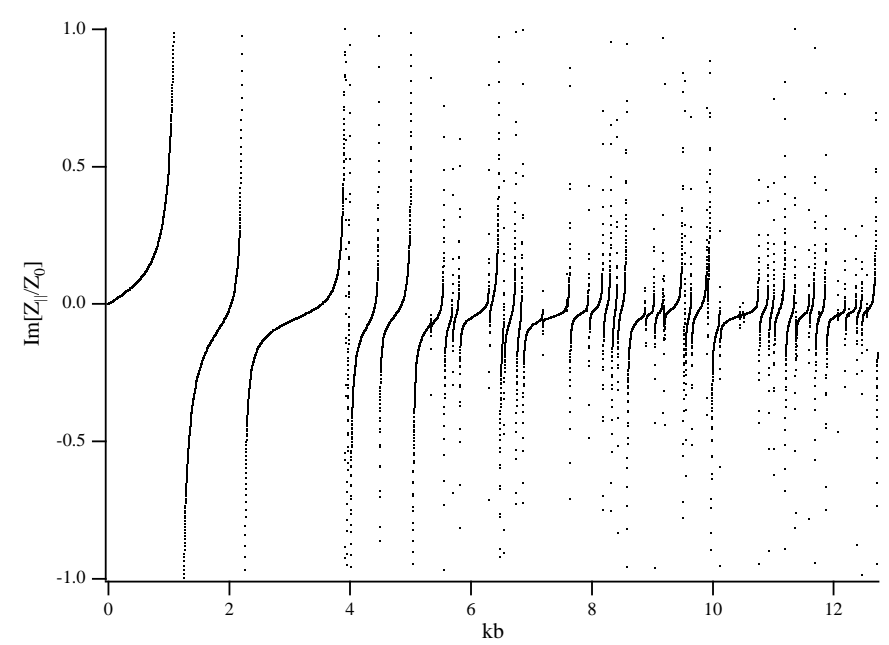

Figure. 1. The imaginary part of longitudinal impedance for $b / a=0.45, L / a=1.1, g / a=0.35$.

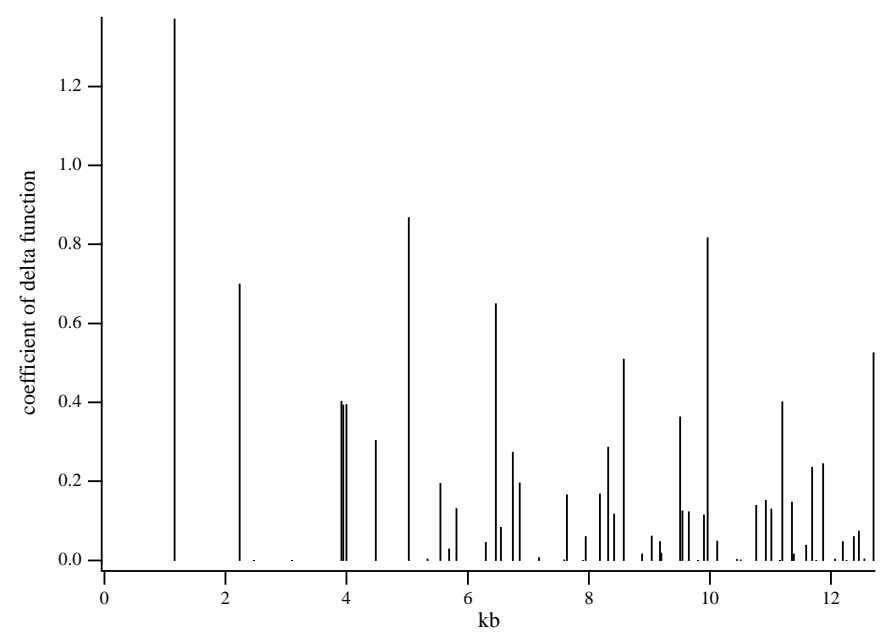

Figure. 2. The real part of longitudinal impedance for $b / a=$ $0.45, L / a=1.1, g / a=0.35$.

$$
\frac{Z_{\perp}(k)}{Z_{0}}=\pi k \sum_{n} b_{n} \delta\left(k^{2}-k_{n}^{2}\right)+j \sum_{n} \frac{b_{n} k_{n}}{k_{n}^{2}-k^{2}},
$$

This time we satisfy $Z_{\perp}(-k)=-Z_{\perp}^{*}(k)$, and the resonances correspond to the propagation of modes proportional to $\cos \theta$, $\sin \theta$, with a phase advance of $k L$ per period. We present the first 10 resonances of the transverse impedance in Table 2.

\section{Acknowledgment}

We are grateful to Dr. Karl L. Bane for providing the output of KN7C and TRANSVRS for our structure.

\section{References}

[1] H. Okamoto, S. Jiang, R. L. Gluckstern, Phys. Rev., E50, 1501(1994).

[2] K. Bane, B. Zotter, in Proceedings of the 11th International Conference on High Energy Accelerators, CERN (Burhäuser Verlag, Basel, 1980), p581.

[3] E. A. Guillemin, Communication Networks, John Wiley \& Sons, Inc., New York, 1931.

[4] The vertical axis is the coefficient of the delta function in Eq. 18.

\begin{tabular}{|r|c|c|}
\hline & $k b$ & $a_{n}\left(Z_{0}\right)$ \\
\hline 1 & 1.16131 & $3.75845 \mathrm{E}-1$ \\
\hline 2 & 2.23213 & $9.99812 \mathrm{E}-2$ \\
\hline 3 & 2.47150 & $1.21422 \mathrm{E}-6$ \\
\hline 4 & 3.10663 & $4.22596 \mathrm{E}-6$ \\
\hline 5 & 3.91728 & $3.27981 \mathrm{E}-2$ \\
\hline 6 & 3.94055 & $3.18982 \mathrm{E}-2$ \\
\hline 7 & 3.99824 & $3.14555 \mathrm{E}-2$ \\
\hline 8 & 4.48055 & $2.16335 \mathrm{E}-2$ \\
\hline 9 & 5.02304 & $5.50932 \mathrm{E}-2$ \\
\hline 10 & 5.33543 & $2.46804 \mathrm{E}-4$ \\
\hline
\end{tabular}

Table. 1. The first 10 resonances of longitudinal impedance for $b / a=0.45, L / a=1.1, g / a=0.35$.

\begin{tabular}{|r|c|c|}
\hline & $k b$ & $b_{n}\left(Z_{0}\right)$ \\
\hline 1 & 1.57045 & $7.10124 \mathrm{E}-1$ \\
\hline 2 & 1.75736 & $3.14108 \mathrm{E}-2$ \\
\hline 3 & 2.31282 & $1.23584 \mathrm{E}-1$ \\
\hline 4 & 2.79476 & $2.20642 \mathrm{E}-2$ \\
\hline 5 & 2.98274 & $1.13804 \mathrm{E}-2$ \\
\hline 6 & 3.24850 & $6.87317 \mathrm{E}-3$ \\
\hline 7 & 3.66906 & $4.27322 \mathrm{E}-2$ \\
\hline 8 & 3.91339 & $3.63055 \mathrm{E}-3$ \\
\hline 9 & 4.08240 & $9.94933 \mathrm{E}-3$ \\
\hline 10 & 4.21653 & $8.24202 \mathrm{E}-3$ \\
\hline
\end{tabular}

Table. 2. The first 10 resonances of transverse impedance for $b / a=0.45, L / a=1.1, g / a=0.35$. 